\title{
Drug/protein interactions studied by time-resolved fluorescence spectroscopy
}

\author{
Thomas Gustavsson*a, Dimitra Markovitsia ${ }^{\mathrm{a}}$, Ignacio Vayáb ${ }^{\text {, Paula Bonancía }}{ }^{\mathrm{b}}$, M.Consuelo Jiménez ${ }^{\mathrm{b}}$, \\ Miguel A. Miranda ${ }^{\mathrm{b}}$ \\ ${ }^{a}$ CNRS, IRAMIS, LIDyL, Francis Perrin Laboratory, URA 2453, 91191 Gif-sur-Yvette, France; \\ ${ }^{\mathrm{b}}$ Departamento de Química/Instituto de Tecnología Química UPV-CSIC, Universitat Politècnica de \\ València, 46022 Valencia, Spain
}

\begin{abstract}
We report here on a recent time-resolved fluorescence study [1] of the interaction between flurbiprofen (FBP), a chiral non-steroidal anti-inflammatory drug, and human serum albumin (HSA), the main transport protein in the human body. We compare the results obtained for the drug-protein complex with those of various covalently linked flurbiprofentryptophan dyads having well-defined geometries. In all cases stereoselective dynamic fluorescence quenching is observed, varying greatly from one system to another. In addition, the fluorescence anisotropy decays also display a clear stereoselectivity. For the drug-protein complexes, this can be interpreted in terms of the protein microenvironment playing a significant role in the conformational relaxation of FBP, which is more restricted in the case of the $(R)$ enantiomer.
\end{abstract}

Keywords: drugs, flurbiprofen, albumin, time-resolved fluorescence, femtosecond, fluorescence upconversion

\section{INTRODUCTION}

The transport and distribution of drugs in the body is a complex mechanisms governed by many different processes. An important role is played by specific transport proteins, responsible for the fixing and release of the drugs. Obtaining a better understanding of the factors that govern the structure and dynamics of drug-protein complexes is therefore of great interest.

Among the various techniques used to study the drug-protein interaction, fluorescence spectroscopy is very useful since it involves the first singlet excited state which is very sensitive to the local surroundings. In particular, time-resolved fluorescence informs on the excited state interactions occurring between the photo-excited drug and the protein, which in turn provides information regarding the structural dynamics of the complex.

Several femtosecond fluorescence spectroscopic measurements of drug-protein interactions have been reported in the past. [2-6]. These have shown the influence of the biological nanocavities not only on the diffusional/orientational motions of the drug, but also on its solvation dynamics and excited state processes such as energy, electron and proton transfer.

We report here on the interaction between flurbiprofen (FBP), a chiral non-steroidal anti-inflammatory drug, and human serum albumin (HSA), the main transport protein in the human body, using time-resolved fluorescence spectroscopy. To this aim, we have used a combination of two time-resolved fluorescence techniques; fluorescence upconversion (FU), with a time-resolution of about 100 femtoseconds, and time-correlated single photon counting (TCSPC), with a timeresolution of about $25 \mathrm{ps}$.

HSA is the most abundant protein in the bloodstream, responsible for the transport of different agents such as drugs. Therefore, the binding of a drug to HSA constitutes a key process, relevant for its distribution. It is known that FBP binds to HSA preferentially in the so-called site II. HSA contains only one tryptophan (W214), situated close to site II. Both FBP and W214 absorb and emit light in the UV spectral region, rendering the analysis of data delicate. FBP can also interact with W214 via energy- or electron-transfer after photo-excitation.

In order to investigate the FBP-Trp interaction in more detail, two covalently linked dyads, $(S, S)$ - or $(R, S)$-, formed by either $(S)$ - or $(R)$-FBP and $(S)$-TrpMe $((S)$-tryptophan methyl ester) have been synthesized and employed as model systems (Figure 1) [7]. Due to their low solubility in water, experiments were performed in acetonitrile. 
Such drug-amino acid dyads have already been designed and studied with success in the past [8,9]. They have provided new mechanistic insight into the fundamental processes that occur between the two chromophores such as energy transfer, electron transfer, exciplex formation, etc.

A

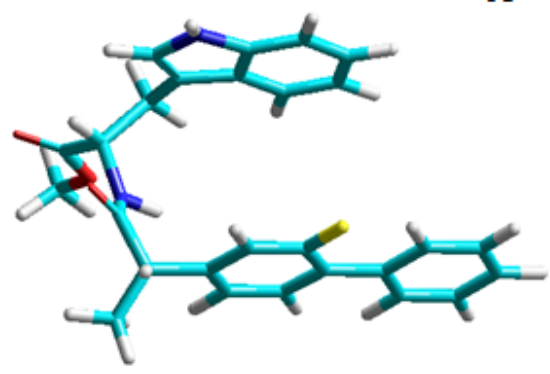

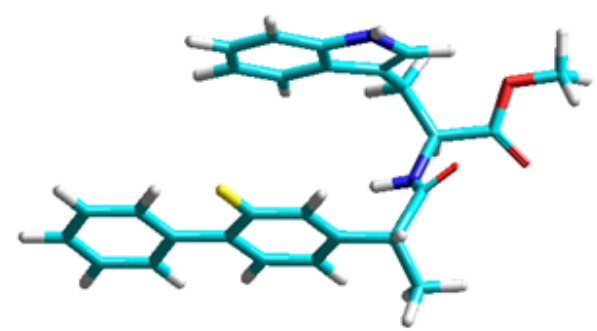

Figure 1. The model dyads $(S, S)$-FBP-TrpMe $(\mathrm{A})$ and $(R, S)$-FBP-TrpMe (B).

\section{STEADY-STATE FLUORESCENCE}

Previous steady-state spectroscopic measurements of the dyads have shown that a strongly stereo-selective fluorescence quenching (>90\%) occurs and that only a weak residual TrpMe emission can be observed (Figure 2A). This has been taken as an indication of efficient FBP $\rightarrow$ Trp energy transfer [2]. For both dyads, a band centered at $450 \mathrm{~nm}$, assigned to exciplex emission, was also observed (dashed lines in Figure 2A).

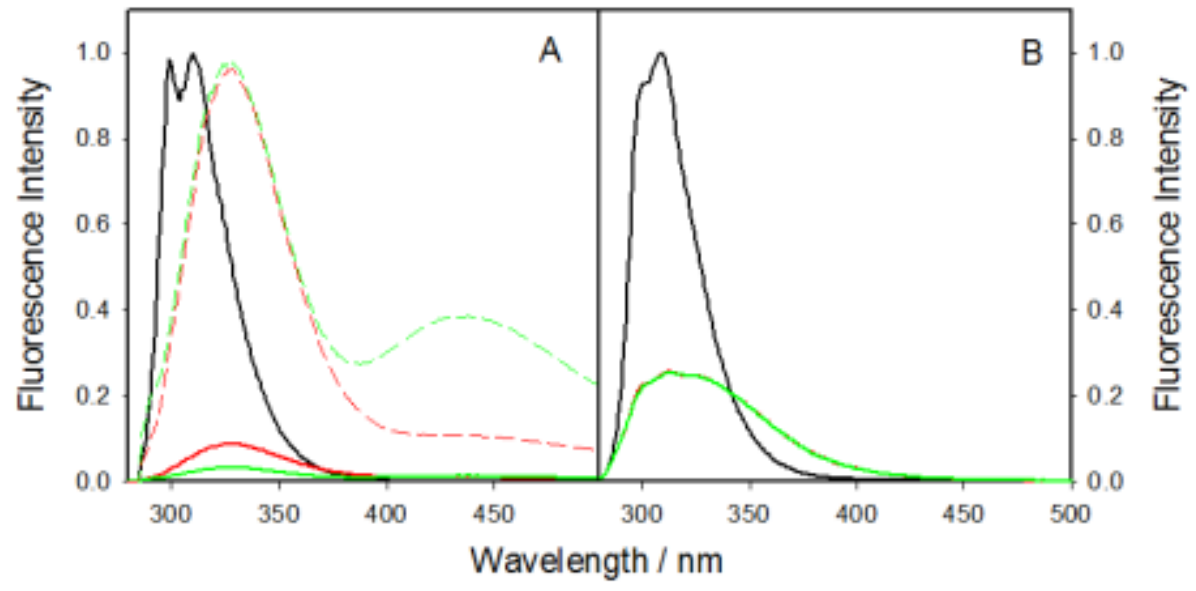

Figure 2. Steady-state fluorescence spectra of (A) $(S)$-FBP (black), $(S, S)$-FBP-TrpMe (red), and $(R, S)$-FBP-TrpMe (green) in acetonitrile. Dashed lines correspond to the normalized emission spectra. (B) (S)-FBP (black), $(S)$-FBP-HSA (red) and

$(R)$-FBP-HSA (green) in PBS, under air, using isoabsorptive solutions at the excitation wavelength $(267 \mathrm{~nm})$.

Strong fluorescence quenching occurs also in the FBP/HSA complex, even though less important and with less marked stereo-differentiation than for the dyads (Figure 2B).

\section{TIME-RESOLVED FLUORESCENCE OF THE DYADS}

Our results show that the fluorescence decays of both dyads are much faster than those of free FBP or TrpMe. At the fluorescence maximum of FBP, around $310 \mathrm{~nm}$, the fluorescence decays of the two dyads recorded by FU occur on the picosecond time scale (Figure 3A). These results are in accordance with a dynamical fluorescence quenching. A significant stereo-selectivity can be observed; the $(R, S)$-dyad emission decaying faster than that of the $(S, S)$-dyad. It 
should be noted that no rise in the Trp fluorescence at $340 \mathrm{~nm}$ could be observed, which would have supported the idea of energy transfer.

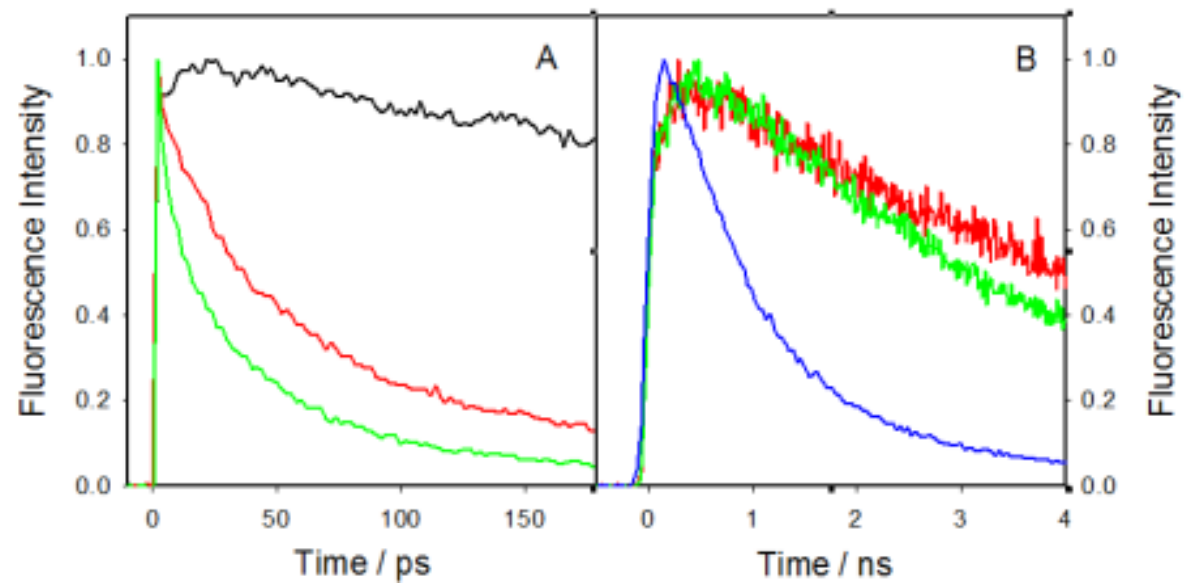

Figure 3. Fluorescence decays at (A) $310 \mathrm{~nm}$ using FU and (B) $450 \mathrm{~nm}$ using TCSPC of (S)-FBP (black), (S,S)-FBP-TrpMe (red), $(R, S)$-FBP-TrpMe (green) and $(S)$-TrpMe (blue) in $\mathrm{MeOH}$.

The fluorescence decays of the two dyads recorded by TCSPC at $450 \mathrm{~nm}$ are much slower than that of TrpMe and are characterized by a rise (Figure 3B), which can be assigned to the formation of the exciplex. Since exciplex formation can be related to charge transfer, this suggests that the fluorescence quenching mechanism also involves electron transfer. Interestingly, both the formation and the decay of the exciplex emission show a clear stereo-differentiation.

\section{TIME-RESOLVED FLUORESCENCE OF THE FBP-HSA COMPLEX}

The time-resolved measurements by FU show that the time evolutions of the fluorescence of $(S)$-FBP/HSA and $(R)$ FBP/HSA are similar at early times (Figure 4A). Only after several tens of picoseconds do the traces start to deviate. Curiously, the stereo-differentiation is inversed with regards to that observed for the dyads, with $(S)$-FBP/HSA showing a faster decay than $(R)$-FBP/HSA.

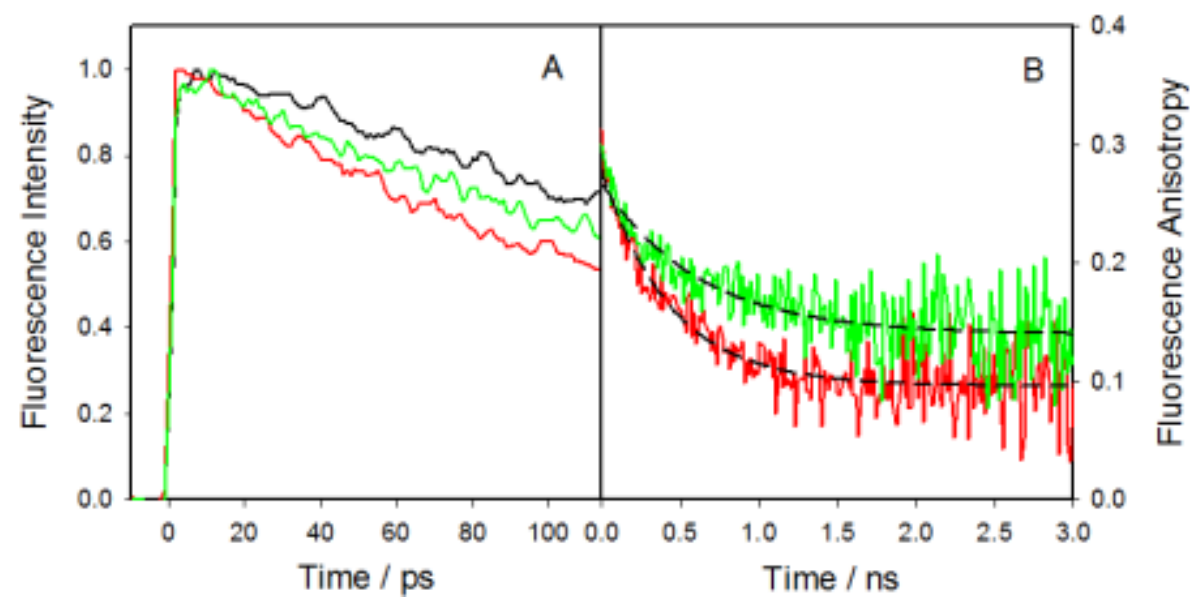

Figure 4. (A) Fluorescence decays (by FU) and (B) fluorescence anisotropy decays (by TCSPC) at $310 \mathrm{~nm}$ of $(S)$-FBP (black), $(S)$-FBP/HSA (red) and ( $R$ )-FBP/HSA (green). (Fitted curves indicated by dashed lines)

There is also a marked stereo-selectivity in the fluorescence anisotropy decays recorded by TCSPC at $310 \mathrm{~nm}$ (Figure 4B). After a fairly rapid decay (hundreds of picoseconds) the anisotropies attain a constant value which persists over several nanoseconds. Interestingly, the fluorescence anisotropy decay of $(S)$-FBP/HSA is faster $(0.44 \pm 0.03 \mathrm{~ns})$ and of higher amplitude than that of $(R)$-FBP/HSA $(0.62 \pm 0.07 \mathrm{~ns})$. 
The constant value at longer times implies a restricted reorientational motion, which can be described by the wobblingin-a-cone model [10]. The different reorientational dynamics observed for the two enantiomers indicate that the conformational relaxation is more restricted for $(R)$-FBP within the binding pocket. This could possibly be related to the pharmaceutical activity of flurbiprofen.

\section{CONCLUSIONS}

The photophysical behaviour of the drug flurbiprofen when covalently linked to Trp in model dyads has been compared with that of its non-covalent complex with human serum albumin.

A very fast dynamic and strongly stereo-selective quenching of the FBP fluorescence is observed in the dyads. This can be attributed to efficient energy and/or electron transfer. The residual Trp fluorescence also shows a stereo-selective quenching, although slower, together with exciplex formation.

Similar trends were observed in the drug-protein complexes, although the kinetics of the involved processes are slower and the stereo-differentiation is opposite to that observed for the dyads. This shows that the relative spatial arrangement of the chromophores constitutes a critical factor in the stereodifferentiation. Moreover, the fluorescence anisotropy clearly showed that the protein microenvironment plays a significant role in the conformational relaxation of FBP, which is more restricted in the case of the $(R)$-enantiomer. This stereo-selectivity is possibly related to the modes of drug binding to the protein, a process of pharmacological relevance.

The present results show that time-resolved fluorescence spectroscopy constitutes a powerful tool to study drug-protein interactions. The combination of two fluorescence techniques, fluorescence upconversion and time-correlated single photon counting, allows to cover a very wide time-window and thereby distinguishing different phenomena. Finally, to our knowledge, this is the first observation of stereo-selective drug-protein interaction dynamics recorded with femtosecond time resolution.

\section{REFERENCES}

[1] Vaya, I., Bonancía, P., Gustavsson, T., Markovitsi, D., Jiménez, M. C. and Miranda, M. A., "Excited state interactions between flurbiprofen and tryptophan in drug/protein complexes and in model dyads. Fluorescence studies from the femtosecond to the nanosecond time domains," Phys. Chem. Chem. Phys., 15, 4727-4734 (2013).

[2] Zhong, D. P., Douhal, A. and Zewail, A. H., "Femtosecond studies of protein-ligand hydrophobic binding and dynamics: Human serum albumin," Proc. Natl. Acad. Sci. U.S.A., 97, 14056-14061 (2000).

[3] Zhong, D., Pal, S. K., Wan, C. and Zewail, A. H., "Femtosecond dynamics of a drug-protein complex: Daunomycin with Apo riboflavin-binding protein," Proc. Natl. Acad. Sci. U.S.A., 98, 11873-11878 (2001).

[4] Kamal, J. K. A., Zhao, L. and Zewail, A. H., "Ultrafast hydration dynamics in protein unfolding: Human serum albumin," Proc. Natl. Acad. Sci. U.S.A., 101, 13411-13416 (2004).

[5] Douhal, A., Sanz, M. and Tormo, L., "Femtochemistry of orange II in solution and in chemical and biological nanocavities," Proc. Natl. Acad. Sci. U.S.A., 102, 18807-18812 (2005).

[6] Cohen, B., Alvarez, C. M., Carmona, N. A., Organero, J. A. and Douhal, A., "Proton-Transfer Reaction Dynamics within the Human Serum Albumin Protein," J. Phys. Chem. B, 115, 7637-7647 (2011).

[7] Vaya, I., Jimenez, M. C. and Miranda, M. A., "Excited-State Interactions in Flurbiprofen-Tryptophan Dyads," J. Phys. Chem. B, 111, 9363-9371 (2007).

[8] Abad, S., Pischel, U. and Miranda, M. A., "Wavelength-dependent stereodifferentiation in the fluorescence quenching of asymmetric naphthalene-based dyads by amines," J. Phys. Chem. A, 109, 2711-2717 (2005).

[9] Abad, S., Vaya, I., Jimenez, M. C., Pischel, U. and Miranda, M. A., "Diastereodifferentiation of novel naphthalene dyads by fluorescence quenching and excimer formation," ChemPhysChem, 7, 2175-2183 (2006).

[10] Schroder, G. F., Alexiev, U. and Grubmuller, H., "Simulation of fluorescence anisotropy experiments: Probing protein dynamics," Biophys. J., 89, 3757-3770 (2005). 